\title{
Bitte des Herausgebers
}

an die Mitglieder der schweizerischen Gesellschaft für Geschichte der Medizin und der Naturwissenschaften und an weitere Kreise, welche sich für die Geschichte der Medizin, Naturwissenschaften und Technik interessieren:

Es ist beabsichtigt, die Zeitschrift «Gesnerus» auch in bibliographischer Hinsicht zu einem Sammelpunkt der naturwissenschafts- und medizingeschichtlichen Forschung der Schweiz auszugestalten. Bereits im nächsten Heft soll mit der Bibliographie begonnen werden. Für den Redaktor ist es bei der sehr weit zerstreuten Literatur unmöglich, selbst bei gewissenhafter Durchsicht der einschlägigen Periodica (ca. 50 schweizerische und einige ausländische) und unter Benutzung der Kataloge der Schweizerischen Landesbibliothek, eine vollständige Übersicht über die literarische Produktion auf diesem Gebiet zu erlangen. Er ist, soll die Bibliographie auf möglichst breiter Basis durchgeführt werden, auf die Mithilfe der Autoren und der Leserschaft, insbesondere derjenigen Leser, welchen regelmäßig ausländische Zeitschriften zur Verfügung stehen, unbedingt angewiesen. Deshalb ergeht die Bitte an alle Autoren: sie möchten ihre einschlägigen Publikationen (Zeitschriftenartikel, biographische Notizen, oder größere Werke) dem Redaktor zur Verfügung stellen, oder wenigstens die genauen Titel (mit Ort, Verlag und Jahr des Erscheinens) bekannt geben. Es betrifft dies alle medizinhistorichen oder naturwissenschaftgeschichtlichen Publikationen, welche seit 1938 in der Schweiz oder im Auslande erschienen sind, letztere insofern es sich um Publikationen handelt, welche die Wissenschaftsgeschichte der Schweiz betreffen, oder von Schweizer Autoren verfaßt sind.

Wenn auch auf diesem Wege keine Vollständigkeit erzielt werden kann, so sollte es doch möglich sein, einen größeren Teil der einschlägigen Veröffentlichungen bibliographisch zu ordnen und diese Bibliographie periodisch in dieser Zeitschrift zu veröffentlichen. Es käme so im Laufe der Jahre eine Bibliographie zustande, welche für Forscher auf diesen Gebieten einige nützliche Vorarbeit leisten könnte. Gleichzeitig würde auch dem interessierten Ausland (ähnlich wie im «Lychnos», dem Jahrbuch der schwedischen Gesellschaft für Geschichte der Naturwissenschaften), die schweizeriche Leistung auf diesem Gebiet bekannt gemacht.

Die der Redaktion zur Verfügung gestellten Publikationen, deren Besprechung im «Gesnerus» vorbehalten bleibt, würden, falls der Autor 
nichts anderes bestimmt, in das Eigentum der Gesellschaft übergehen und in die im Entstehen begriffene medizinhistorische und naturwissenschaftsgeschichtliche Bibliothek derselben eingeordnet, deren Katalog periodisch zur Veröffentlichung gelangen wird. Diese Bibliothek steht den Mitgliedern unserer Gesellschaft nach einem noch aufzustellenden Reglement zur freien Benutzung zur Verfügung.

Einsendungen für die Bibliographie und Bibliothek sind an den Unterzeichneten (Pharmakologisches Institut der Universität, Gloriastraße 32, Zürich), mit bestem Dank, erbeten.

H. Fischer.

\section{Zur Geschichte der Wissenschaften im Allgemeinen}

Die Geschichte der Wissenschaften ist eine große Fuge, in der die Stimmen der Völker nach und nach zum Vorschein kommen.

Wenn man die Probleme des Aristoteles ansieht, so erstaunt man über die Gabe des Bemerkens und für was alles die Griechen Augen gehabt haben. Nur begehen sie den Fehler der Übereilung, da sie von dem Phänomen unmittelbar zur Erklärung schreiten, wodurch denn ganz unzulängliche theoretische Aussprüche zum Vorschein kommen. Dieses ist jedoch der allgemeine Fehler, der noch heutzutage begangen wird.

Um sich aus der grenzenlosen Vielfachheit, Zerstückelung und Verwicklung der modernen Naturlehre wieder ins Einfache zu retten, muß man sich immer die Frage vorlegen: wie würde sich Plato gegen die Natur, wie sie uns jetzt in ihrer größern Mannigfaltigkeit, bei aller gründlichen Einheit, erscheinen mag, benommen haben?

Man rühmt das achtzehnte Jahrhundert, daß es sich hauptsächlich mit Analyse abgegeben; dem neunzehnten bleibt nun die Aufgabe, die falschen obwaltenden Synthesen zu entdecken und deren Inhalt aufs neue zu analysieren.

Gœthe

Aus den Aphorismen zur Wissenschaftsgeschichte. 